\title{
Extranodal Rosai-Dorfman Disease with Cutaneous and Periodontal Involvement: A Rare Presentation
}

\author{
M.J. Molina-Garrido ${ }^{a} \quad$ C. Guillén-Ponce ${ }^{b}$ \\ Departments of Medical Oncology, aHospital General Virgen de la Luz, Cuenca, \\ and ${ }^{b}$ Hospital Ramón y Cajal, Madrid, Spain
}

\section{Key Words}

Cervical lymphadenopathy · Chemotherapy · Cutaneous disease · Histiocytosis . Rosai-Dorfman disease

\begin{abstract}
Sinus histiocytosis with massive lymphadenopathy (SHML) or Rosai-Dorfman disease (RDD) was first described as a distinct clinicopathological entity by Rosai and Dorfman in 1969. SHML is commonly characterized by painless cervical lymphadenopathy, and its clinical course is generally benign and self-limiting. This disorder commonly involves the lymph nodes but may secondarily involve the skin. However, purely cutaneous disease without lymphadenopathy or internal organ involvement rarely occurs. In the absence of the massive lymphadenopathy that is characteristic of RDD, the diagnosis of purely cutaneous RDD may be complicated by the rare, nonspecific clinical appearance of skin lesions and the broad histopathological differential diagnosis of this disorder. A high level of suspicion of this disease on the part of the clinician or pathologist is often required. We present a case of cutaneous RDD associated with involvement of periodontal tissue.
\end{abstract}

\section{Case Report}

A 43-year-old man with a past medical history of Wolff-Parkinson-White syndrome presented with a 2-month history of an asymptomatic parieto-occipital skin lesion (a 1.5-cm red-yellow nodule). Superficial lymph nodes were not enlarged. The skin lesion was removed. The microscopic examination revealed numerous histiocytes with abundant pale cytoplasm invading the lymph sinuses. Occasionally, engulfed lymphocytes were noted in the cytoplasm of the histiocytes. Immunological staining showed that the invading histiocytes were positive for CD68 and S-100 protein and were negative for CD1a and CD20. These findings were consistent with the characteristics of Rosai-Dorfman disease (RDD). The leukocyte count was normal. No anemia or hypergammaglobulinemia was noted, and there was no reversal of $\mathrm{CD} 4 / \mathrm{CD} 8$. The tests for hepatitis B surface antigen and antibodies to hepatitis $\mathrm{C}$ virus were negative. Neither antibodies to human T-cell leukemia virus 1 or human immunodeficiency virus nor 
Epstein-Barr virus RNA were observed. Thoracic and abdominal CT showed a normal lung field with no mediastinal lymphadenopathy, no hepatosplenomegaly and no large lymphadenopathy.

Hemophagocytic cells were absent in the bone marrow. The patient was eventually diagnosed with sinus histiocytosis with massive lymphadenopathy involving the skin. Because he also described a 1-year history of pain in his right inferior maxillary area, a positron emission tomography (PET) scan was performed, which showed fluorodeoxyglucose uptake in the right inferior maxillary area (SUV 8.7). Histological examination of the mucosal biopsy specimen showed large histiocytic cells with small round-to-oval nuclei, which were strongly positive for S-100 and CD68, without emperipolesis. The lesion was completely removed during surgery. A repeat PET was normal. The patient remained asymptomatic with no signs of recurrence at the 3-month follow-up.

\section{Discussion}

Sinus histiocytosis with massive lymphadenopathy (SHML) is a rare disorder that is characterized by non-malignant proliferation of distinctive histiocytic/phagocytic cells within lymph node sinuses and the lymphatic system in extranodal sites. This condition primarily affects children and young adults. In the literature, 423 cases of RDD have been reported [1].

Although SHML is relatively rare, purely cutaneous RDD is even less common, accounting for approximately $3 \%$ of SHML cases in one large study [2]. SHML skin lesions may be diverse and can be found in virtually any location, including the face, ears, trunk, extremities or genitalia $[1,3]$. Several authors have suggested that cutaneous RDD is a distinct clinical entity because of its unique epidemiology and the lack of systemic involvement even with long-term follow-up [3-5]. The characteristics of nodal and purely cutaneous RDD are shown in table 1.

Extranodal disease is documented in $30-40 \%$ of patients, and in some patients it presents without associated lymphadenopathy, which may develop later in the course of disease [1]. Extranodal involvement of at least one site is not uncommon, and it is rarely the initial and only manifestation of the disease. The most commonly affected extranodal sites include the skin and soft tissue, the upper respiratory tract and bone followed by the genitourinary tract, the lower respiratory tract, the oral cavity, the gastrointestinal tract [6], the orbit [7] and the testes. In cutaneous RDD, extranodal disease may also develop at other sites, e.g. the eye, most commonly leading to uveitis $[8,9]$. Central nervous system involvement is very rare $(<5 \%)[10]$.

Clinical laboratory findings include hematologic abnormalities, such as normocytic or microcytic anemia $[1,11]$ and polyclonal hypergammaglobulinemia (90\%). Rarely, patients exhibit rheumatoid factor, antinuclear antibodies and a reversal of the CD4/CD8 ratio among peripheral lymphocytes. Immunological abnormalities are found in a significant number of patients and often lead to a clinically unfavorable disease course. Consistent serologic findings are lacking, although antibodies to pathogens, including EBV and human herpesvirus (HHV-6), have been reported [12]. A small subset of patients also has concurrent neoplasia, including non-Hodgkin lymphoma, other histiocytic proliferations, myeloma, melanoma and carcinoma. In the case presented herein, there was no relation to EBV, HIV or HHV-6 infection, or to any other neoplasia.

The hallmark of the SHML histiocyte is the presence of a variable number of intact lymphocytes within the cytoplasm of the cell, which is a phenomenon referred to as lymphophagocytosis or emperipolesis [1]. The histological differential diagnosis includes 
Langerhans cell histiocytosis, histiocytic sarcoma, storage disorders such as Gaucher's disease, classical Hodgkin's lymphoma, metastatic melanoma and carcinoma, as well as infections caused by Histoplasma and mycobacteria that involve the lymph nodes.

The most useful immunohistological markers for SHML histiocytes are the S-100 protein and the pan-macrophage antigens (CD86, CD14, CD15 and CD64). Whereas Langerhans cells positively stain for the S-100 protein and CD1a, only rare CD1a-positive cells are found in SHLM.

The clinical course of SHML is characterized by spontaneous resolution in most cases, especially in those presenting with localized lymph node involvement. Sometimes, there are episodes of exacerbation alternating with periods of remission that continue for many years. Persistence of lymphadenopathy or progression to widespread dissemination may occur, particularly in SHML that involves the kidneys, the lower respiratory tract or the liver with associated immunological dysfunction. The reported mortality rate is $7 \%$, with many patients who have a fatal outcome having concomitant immune dysfunction [13].

The pathogenesis of this disease is poorly understood, although some researchers have suggested that either infection or immunodeficiency might play a role [14]. Recently, cases of SHML associated with the autoimmune lymphoproliferative syndrome have been described [15].

The disease is self-limiting in most cases. In a recent review of treatment strategies for SHML, $50 \%$ of patients $(40 / 80)$ did not require any treatment. Patients given antibiotics or anti-tuberculous drugs showed no response. Steroid therapy resulted in a reduction in lymphadenopathy and associated fever. Only 2 of a total of 12 patients achieved complete and durable remission after treatment with methotrexate. High-dose interferon- $\alpha$ was given to 1 patient, which resulted in long-term remission [16]. In a recent report, the use of the purine analogue 2-chlorodeoxyadenosine was found to be of clinical utility in these cases [17]. The ideal treatment for SHML has not yet been determined. Cutaneous lesions have been reported to respond to radiotherapy [4], cryotherapy, excision [4, 5], topical and oral corticosteroids [4], and high-dose thalidomide [5].

The diagnosis of RDD should be considered in any atypical chronic inflammatory lesion with a histiocytic component involving multiple organ systems, especially the skin. In our case, the inflammatory lesion was localized to the maxillary area.

Immunohistochemical stains for the S-100 protein should be performed in these cases. To our knowledge, this is the first case reported with such characteristics. 
Table 1. Differential characteristics of nodal and purely cutaneous Rosai-Dorfman disease

\begin{tabular}{|c|c|c|}
\hline Characteristics & Nodal Rosai-Dorfman & Purely cutaneous Rosai-Dorfman \\
\hline Frequency & $60-70 \%$ & $3 \%$ \\
\hline Predominant sex & male & female \\
\hline Age & 20.6 years & 43.7 years \\
\hline Race & no race predominant & Asian/white \\
\hline Presenting symptoms & painless lymphadenopathy & cutaneous lesion \\
\hline General symptoms & $\begin{array}{l}\text { typically fever, malaise, } \\
\text { night sweats }\end{array}$ & usually none \\
\hline Nodal involvement & yes & no \\
\hline Involved area & cervical region $(90 \%)$ & face, ears, trunk, extremities, genitalia \\
\hline \multicolumn{3}{|l|}{ Biopsy } \\
\hline Emperipolesis & very common & not very common \\
\hline Fibrosis & not very common & very common \\
\hline S-100 & positive & positive \\
\hline CD1a & negative most times & usually negative \\
\hline \multirow[t]{6}{*}{ Treatment } & observation & observation \\
\hline & steroids & steroids (oral or local) \\
\hline & chemotherapy & radiotherapy \\
\hline & methotrexate & cryotherapy \\
\hline & alkylating agents & surgery \\
\hline & high doses of interferon- $\alpha$ & high doses of thalidomide \\
\hline
\end{tabular}

\section{References}

1 Foucar E, Rosai J, Dorfman R: Sinus histiocytosis with massive lymphadenopathy (Rosai-Dorfman disease): review of the entity. Semin Diagn Pathol 1990;7:19-73.

2 Chuah KL, Tan PH, Hwang SG, Ong BH: Cutaneous Rosai-Dorfman disease - a cytopathologic review of 2 cases. Singapore Med J 2000;41:122-125.

-3 Wang KH, Chen WY, Liu HN, Huang CC, Lee WR, Hu CH: Cutaneous Rosai-Dorfman disease: clinicopathological profiles, spectrum and evolution of 21 lesions in six patients. Br J Dermatol 2006;154:277286.

4 Brenn T, Calonje E, Granter SR, et al: Cutaneous Rosai-Dorfman disease is a distinct clinical entity. Am J Dermatopathol 2002;24:385-391.

5 Lu C, Kuo T, Wong W, Hong H: Clinical and histopathologic spectrum of cutaneous Rosai-Dorfman disease in Taiwan. J Am Acad Dermatol 2004;51:931-939.

6 Alatassi H, Ray MB, Galandiuk S, Sahoo S: Rosai-Dorfman disease of the gastrointestinal tract: report of a case and review of the literature. Int J Surg Pathol 2006;14:95-99.

7 Wang E, Anzai Y, Paulino A, Wong J: Rosai-Dorfman disease presenting with isolated bilateral orbital masses: report of two cases. Am J Neuroradiol 2001;22:1386-1388.

-8 Salim A, Williamson M, Barker F, Hughes J: Steroid responsive cutaneous Rosai-Dorfman disease associated with uveitis and hypothyroidism. Clin Exp Dermatol 2002;27:277-279.

-9 Kroumpouzos G, Demierre MF: Cutaneous Rosai-Dorfman disease: histopathological presentation as inflammatory pseudotumor. A literature review. Acta Derm Venereol 2002;82:292-296.

10 Wu M, Anderson AE, Kahn LB: A report of intracranial Rosai-Dorfman disease with literature review. Ann Diagn Pathol 2001;5:96-102.

11 Grabczynska SA, Toh CT, Francis N, Costello C, Bunker CB: Rosai-Dorfman disease complicated by autoimmune haemolytic anaemia: case report and review of a multisystem disease with cutaneous infiltrates. Br J Dermatol 2001;145:323-326. 
12 Luppi M, Barozzi P, Garber R, et al: expression of human herpesvirus-6 antigens in benign and malignant lymphoproliferative diseases. Am J Pathol 1998;153:815-823.

13 Komp DM: The treatment of sinus histiocytosis with massive lymphadenopathy (Rosai-Dorfman disease). Semin Diagn Pathol 1990;7:83-86.

14 Alvarez Alegret R, Martinez Tello A, Ramirez T, Gallego P, Martinez D, Garcia Julian G: Sinus histiocytosis with massive lymphadenopathy (Rosai-Dorfman disease). Diagnosis with fine-needle aspiration in a case with nodal or nasal involvement. Diagn Cytopatol 1995;13:333-335.

15 Maric I, Pittaluga S, Dale J, Straus SE, Jaffe ES: Sinus histiocytosis with massive lympadenopathy in patients with autoimmune lymphoproliferative syndrome. Mode Pathol 2004;17:258A.

16 Pulsoni A, Anghel G, Falcucci P, et al: Treatment of sinus histiocytosis with massive lymphadenopathy (RosaiDorfman disease): report of a case and literature review. Am J Hematol 2002;69:67-71.

$\checkmark 17$ Rodríguez-Galindo C, Helton KJ, Sanchez ND, Rieman M, Jeng M, Wang W: Extranodal Rosai-Dorfman disease in children. J Pediatr Hematol Oncol 2004;26:19-24. 